\title{
Two small regulatory RNAs establish opposing fates of a developmental axis
}

\section{Fabio T.S. Nogueira, ${ }^{1,3}$ Shahinez Madi, ${ }^{1,3}$ Daniel H. Chitwood, ${ }^{1,2}$ Michelle T. Juarez, ${ }^{1,4}$ and Marja C.P. Timmermans ${ }^{1,2,5}$}

\begin{abstract}
${ }^{1}$ Cold Spring Harbor Laboratory, Cold Spring Harbor, New York 11724, USA; ${ }^{2}$ Watson School of Biological Sciences, Cold Spring Harbor, New York 11724, USA
\end{abstract}

Small RNAs are important regulators of gene expression. In maize, adaxial/abaxial (dorsoventral) leaf polarity is established by an abaxial gradient of microRNA166 (miR166), which spatially restricts the expression domain of class III homeodomain leucine zipper (HDZIPIII) transcription factors that specify adaxial/upper fate. Here, we show that leafbladeless1 encodes a key component in the trans-acting small interfering RNA (ta-siRNA) biogenesis pathway that acts on the adaxial side of developing leaves and demarcates the domains of hd-zipIII and miR166 accumulation. Our findings indicate that tasiR-ARF, a ta-siRNA, and miR166 establish opposing domains along the adaxial-abaxial axis, thus revealing a novel mechanism of pattern formation.

Supplemental material is available at www.genesdev.org.

Received January 8, 2007; revised version accepted February 20, 2007.

In both animals and plants, many developmentally important regulatory genes are predicted targets of microRNAs (miRNAs), which suggests that such small RNAs constitute a class of developmental determinants (Alvarez-Garcia and Miska 2005; Jones-Rhoades et al. 2006). Patterning and outgrowth of lateral organs in plants depend on the specification of adaxial/abaxial (dorsoventral) polarity in the incipient primordium. This asymmetry is established through the polarized expression of class III homeodomain leucine zipper (HD-ZIPIII) transcription factors that specify adaxial/upper cell fate (McConnell et al. 2001; Emery et al. 2003; Juarez et al. 2004a). The adaxial-specific expression of hd-zipIII family members is delineated by the expression pattern of a 21-nucleotide (nt) miRNA, miR166, which directs the cleavage of $h d$-zipIII transcripts (Juarez et al. 2004a; Kidner and Martienssen 2004). In maize, miR166 accumulates most abundantly immediately below the incipient leaf, but a gradient of miR166 extends into the abaxial side of the initiating organ that establishes organ polarity (Juarez et al. 2004a).

[Keywords: Meristem; leaf; polarity; pattern formation; microRNA trans-acting siRNA

${ }^{3}$ These authors contributed equally to this work.

${ }^{4}$ Present address: Cell and Developmental Biology, University of California, San Diego, 9500 Gilman Drive, La Jolla, CA 92093, USA

${ }^{5}$ Corresponding author.

E-MAIL timmerma@cshl.edu; FAX (516) 367-8369.

Article is online at http://www.genesdev.org/cgi/doi/10.1101/gad.1528607.
Specification of adaxial/abaxial organ polarity in maize also requires the activity of leafbladeless1 (1bl1). Recessive mutations in $1 b 11$ lead to a variable abaxialization of leaves (Timmermans et al. 1998). The weak lbl1-ref allele causes a partial loss of adaxial identity revealed as patches of abaxial cells on the upper leaf surface, whereas leaves of the severe ragged seedling1 allele (1bl1-rgd1) are often radially symmetric and completely abaxialized (Fig. 1A). Expression of the hd-zipIII family member rld1 is reduced in $1 b 11$ mutants. Conversely, increased levels of hd-zipIII expression in Rld1-O mutants, which carry a miR166-insensitive allele of rld1, can fully suppress the vegetative defects of $1 b l 1$ (Juarez et al. 2004b). Ibl1 thus contributes to organ polarity by regulating the accumulation of rld1 transcripts on the adaxial side of the developing leaf.

Here, we show that $1 b l 1$ encodes a homolog of SUPPRESSOR-OF-GENE-SILENCING3 (SGS3), which is specifically required for the biogenesis of trans-acting small interfering RNAs (ta-siRNAs) (Peragine et al. 2004; Vazquez et al. 2004). Ta-siRNAs are derived from noncoding TAS transcripts that are initially targeted for cleavage by specific miRNAs (Allen et al. 2005). However, unlike most miRNA-directed cleavage products, TAS cleavage fragments are converted into doublestranded RNAs through the activities of SGS3 and RNADEPENDENT RNA POLYMERASE6 (RDR6), and subsequently processed by DICER-LIKE4 (DCL4) into 21base-pair (bp) siRNAs that guide the cleavage of target transcripts, similar to the action of miRNAs (Allen et al. 2005; Gasciolli et al. 2005; Xie et al. 2005; Yoshikawa et al. 2005).

The Arabidopsis ta-siRNAs, ta-siR2141, and tasiR2142 (referred to hereafter as tasiR-ARF), regulate expression of the AUXIN RESPONSE FACTOR (ARF) genes ARF3 and ARF4 (Allen et al. 2005; Williams et al. 2005). These transcription factors act redundantly to promote abaxial fate, inferring a role for the ta-siRNA pathway in leaf polarity (Pekker et al. 2005). However, a role for ta-siRNAs in leaf polarity remains tentative, as Arabidopsis mutants that block ta-siRNA biogenesis, such as sgs3, develop no obvious leaf polarity defects (Peragine et al. 2004; Adenot et al. 2006; Hunter et al. 2006). Here, we demonstrate an essential role for the maize ta-siRNA pathway in the specification of adaxial fate. We show that tasiR-ARF accumulates on the adaxial side of developing leaf primordia and restricts the expression domain of abaxial determinants, including miR166. As miR166 defines the abaxial side of the leaf, our results reveal a novel mechanism of pattern formation in which asymmetry along a developmental axis is determined by two opposing small RNAs.

\section{Results and Discussion}

Members of the $h d$-zipIII family contribute redundantly to adaxial fate (Emery et al. 2003; Juarez et al. 2004b). To assess whether loss of adaxial identity in $l b 11$ is associated with altered expression of hd-zipIII family members other than rld1, we compared the expression pattern of rolled leaf2 (rld2) in wild-type and lbl1-rgd1 mutant apices. rld2 is normally expressed at the tip of the shoot apical meristem (SAM), in the vasculature, and along the adaxial side of developing leaf primordia (Fig. 1B). This 


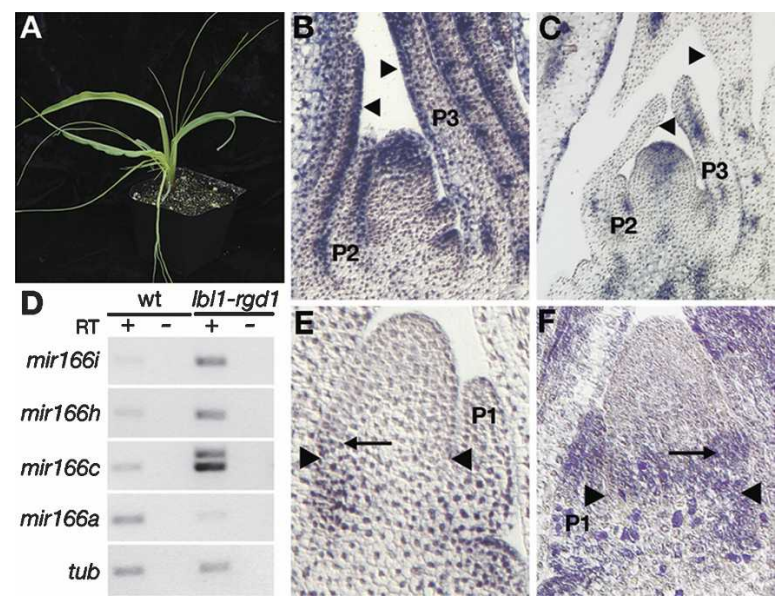

Figure 1. leafbladeless1 (1bl1) establishes leaf polarity by demarcating the domains of $h d$-zipIII and miR166 expression. (A) lbl1rgd1 seedling with thread-like abaxialized leaves. $(B)$ Longitudinal section through a wild-type apex shows rld2 expression in the SAM, vasculature, and adaxially in leaf primordia (arrowheads). (C) In $1 b 11$ rgd1, meristematic, and adaxial (arrowheads) rld2 expression is reduced. $(D)$ Precursor levels for mir166i, mir166h, and mir166c are increased in $1 b 11$-rgd1 compared with wild type, whereas expression of mir166a is reduced. (E) In wild type, miR166 is expressed below and on the abaxial side of the incipient leaf (arrow). (F) In lbl1-rgd1, miR166 is expressed at the base of the SAM and uniformly throughout the incipient (arrow) and P1 primordia. Arrowheads in $E$ and $F$ mark the base of the incipient leaf.

expression pattern resembles that of $r l d 1$ and supports a redundant role for these hd-zipIII genes in promoting adaxial fate. rld2 expression in the vasculature is mostly unaffected in $1 b 11$-rgd1, but the levels of rld2 transcripts in the SAM and on the adaxial leaf surfaces are dramatically reduced or lost in 1bl1-rgd1 (Fig. 1C). These observations indicate that $1 b l 1$ contributes to leaf polarity by regulating the adaxial expression of multiple hd-zipIII genes. Perhaps $1 b 11$ regulates $h d$-zipIII expression at the transcriptional level; alternatively, because the $h d$-zipIII expression domain in the leaf is defined by miR166 (Juarez et al. 2004a; Timmermans et al. 2004), $1 b 11$ could affect hd-zipIII expression through changes in the pattern of miR166 accumulation. To test this possibility, we analyzed expression of miR166 in 1b11-rgd1.

The maize genome includes at least nine loci, mir166a to mir166i, with the potential to generate identical copies of the mature miR166. We used RT-PCR to examine the effect of $1 b 11$ on mir166 precursor levels in vegetative apices comprising the SAM and four-leaf primordia. Expression levels for mir166c, mir166h, and mir166i are increased in 1bl1-rgd1 as compared with wild type, whereas transcript levels for mir166a are reduced in $1 b 11$ rgd1 (Fig. 1D). Expression levels of the remaining mir166 family members are unaltered in $1 b 11$-rgd1, indicating that $1 b 11$ affects the accumulation of a specific subset of mir166 precursors.

We subsequently used in situ hybridization to determine whether the changes in mir166 transcript levels associated with reduced LBL1 activity also affect the pattern of miR166 accumulation. No hybridization signal was detected using a mir166a precursor-specific probe that excludes the mature miRNA (Supplementary Fig. 1). However, consistent with previous results (Juarez et al. 2004a), in situ hybridization with a probe complemen- tary to the mature miR166 showed that the highest concentration of miR166 occurs immediately below the incipient leaf and that miR166 accumulates in a graded pattern on the abaxial side of the initiating organ (Fig. 1E). Expression of miR166 in the region below the incipient leaf is unaffected in 1b11-rgd1, but miR166 expression at the site of leaf initiation is markedly changed in this mutant (Fig. 1F). In 1b11-rgd1, miR166 is ectopically expressed in a ring at the base of the SAM that overlaps broadly with the incipient leaf. Expression of miR166 in the P1 primordium also persists in a broader domain and includes both the abaxial and adaxial sides. These findings are consistent with the decrease in hdzipIII expression in $1 b 11$ leaf primordia and indicate a role for $1 b 11$ in promoting adaxial and/or restricting abaxial fate. They also support a distinct contribution of $1 b 11$ to hd-zipIII expression in the tip of the SAM (Juarez et al. 2004b).

To gain insight into the mechanism with which $1 b 11$ contributes to leaf polarity, we used a directed transposon-tagging strategy to clone $1 b 11$. One novel allele, $1 b 11$ 54 , was recovered that conditions a weak mutant phenotype. Genetic analysis identified a single Mu8 transposable element that cosegregates with the 1bl1-54 mutation $(<0.2 \mathrm{cM})$ and is absent in the progenitor lines. This Mur element had inserted into the first intron of a gene encoding a protein with high homology with SGS3 (Fig. 2A,B; Mourrain et al. 2000). The maize and Arabidopsis proteins share $65 \%$ amino acid similarity overall, but the degree of sequence similarity is higher in the Zn-finger (92\%) and XS domains (79\%) that define the SGS3 family of proteins (Fig. 2B). Consistent with its mild phenotypic effects, the 1b11-54 insertion allele has a moderate effect on normal transcript levels (Fig. 2C). Sequence analysis of additional mutant alleles confirmed that $1 b 11$ encodes a SGS3 homolog (Fig. 2A). We found that a single base-pair change in a second intron splice site that reduces the level of normally spliced transcripts distinguishes the weak 1bl1-ref allele from its progenitor (Fig. 2A,C). 1b11-rgd1 as well as the severe 1b11-372 allele result from point mutations that affect $1 b 11$ expression and cause amino acid substitutions at critical residues of the Zn-finger (Fig. 2A-C; Supplementary Fig. 2).

SGS3 is required for the biogenesis of ta-siRNAs upon miRNA-directed cleavage of TAS precursors (Peragine et al. 2004; Vazquez et al. 2004; Allen et al. 2005). Three gene families that generate ta-siRNAs are known in Arabidopsis. TAS1 and TAS2 precursors are targets of miR173, whereas production of ta-siRNAs from TAS3 depends on cleavage by miR390 (Allen et al. 2005). Database analysis identified no maize homologs of TAS1, TAS2, and MIR173, but the maize genome includes one mir390 locus and four TAS3 homologs, tas3a-tas3d, whose transcripts are predicted targets for miR390 (Fig. 3A). RT-PCR analysis showed that all four tas3 genes as well as mir390 are expressed in vegetative apices (Fig. 3B). Only subtle differences in the levels of tas3 transcripts were observed in $1 b 11-r g d 1$ as compared with wild type, but primary transcripts from mir390 are clearly elevated in 1bl1-rgd1 (Fig. 3B). Because mir390 is in turn required for ta-siRNA biogenesis, this suggests that negative feedback regulation may exist in the ta-siRNA pathway of maize. Perhaps mir390 expression is under control of a ta-siRNA target gene.

Ta-siRNAs are generated with a 21-nt phasing starting at the miRNA cleavage site (Fig. 3A; Allen et al. 2005). 
A

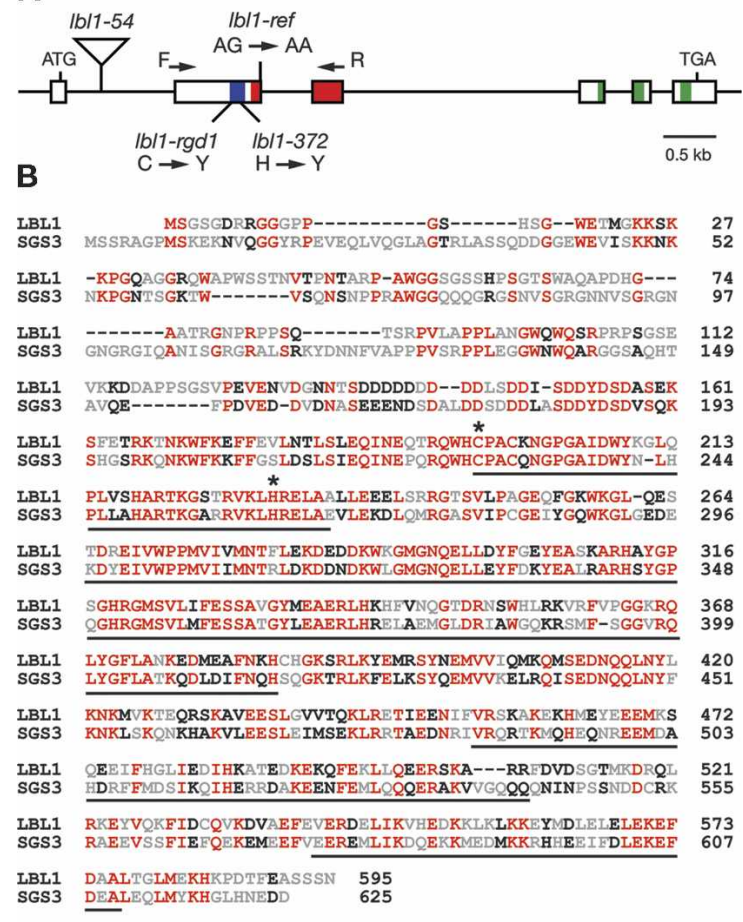

c

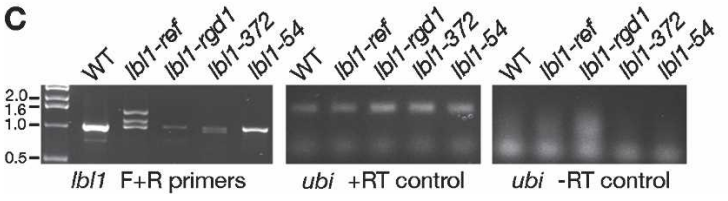

Figure 2. $1 b 11$ encodes a homolog of SUPPRESSOR-OF-GENESILENCING3 (SGS3). (A) Diagrammatic representation of the $1 b 11$ gene. Boxes represent exons, and the zinc-finger, XS, and coil-coiled domains are highlighted in blue, red, and green, respectively. $\mathrm{F}$ and $\mathrm{R}$ represent primers used for RT-PCR analysis in C. Mutations in four mutant 1 lbl1 alleles are indicated. (C) Cysteine; $(\mathrm{H})$ histidine; $(\mathrm{Y})$ tyrosine. $(B)$ Alignment of the maize LBL1 and Arabidopsis SGS3 proteins, with red indicating identity and black indicating similarity. The zinc-finger, XS, and coil-coiled domains are underlined, and asterisks mark the amino acids mutated in $1 b 11$-rgd1 and $1 b 11-372$. (C) $1 b 11$ transcript levels are reduced in the mutants compared with wild type. Loading and -RT controls are shown.

Consequently, their sequences as well as potential targets can be predicted using computational approaches. Despite extensive sequence divergence between the Arabidopsis and maize TAS3 genes, the potential for tas 3 transcripts to yield copies of tasiR-ARF is conserved in maize. tas $3 a-t a s 3 c$ contain two tandemly arranged copies of tasiR-ARF, whereas tas $3 d$ contains a single tasiRARF sequence (Fig. 3A). To establish that LBL1 functions in the biogenesis of ta-siRNAs, we analyzed the accumulation of tasiR-ARF in both wild-type and 1bl1-rgd1 apices (Fig. 3C). Because ta-siRNAs accumulate to unusually low levels in comparison to most miRNAs (Allen et al. 2005; Lu et al. 2006), small RNA blots were hybridized with a 16-nt LNA-modified DNA probe that recognizes tasiR-ARF derived from each of the tas3 loci (Fig. 3A). tasiR-ARF accumulates to detectable levels only in wild-type apices, suggestive of functional conservation between LBL1 and SGS3 (Fig. 3C).

To substantiate a role for $1 b 11$ in ta-siRNA biogenesis, we also tested whether loss of LBL1 activity affects the cleavage of ta-siRNA target transcripts. Prospective targets were identified for tasiR-ARF only, and as in Arabidopsis, these include four members of the maize arf3 gene family, referred to herein as arf3a-arf3d. We used 5' RACE to validate arf3a as a tasiR-ARF target and to determine the effect of mutations in $1 b 11$ on ta-siRNAdirected arf3a cleavage. arf3a contains two tasiR-ARF complementary sites (A and B). RNA from wild-type apices yielded one predominant 5' RACE product for arf $3 a$, resulting from cleavage at positions 9 or 10 of the tasiRARF complementary site B (Fig. 3D,E). Importantly, the abundance of this $5^{\prime}$ RACE product is strongly reduced in $1 b 11$-rgd1 (Fig. 3D), whereas arf3a transcript levels are increased in $1 b 11$-rgd1 apices compared with wild type (Fig. 3B). These data indicate that arf3a is a direct target of tasiR-ARF and reveal extensive conservation in the ta-siRNA pathway between maize and Arabidopsis.

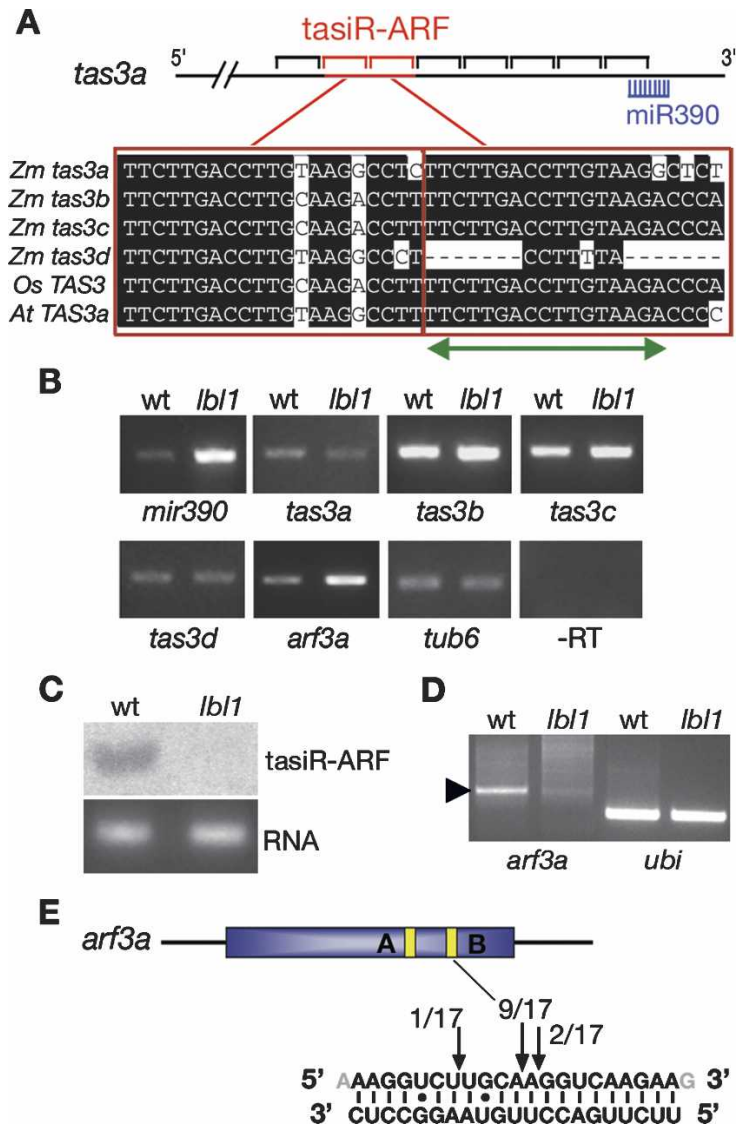

Figure 3. $1 b l 1$ encodes an essential component of the ta-siRNA pathway. (A) Diagram of the tas3a transcript. The 21-nt intervals phased relative to the miR390 cleavage site are shown as black brackets and the red brackets indicate the positions of tasiR-ARF. An alignment of tasiR-ARF sequences from maize, rice, and Arabidopsis is shown below, and the green arrow indicates complementarity to the tasiR-ARF LNA probe. Note that tas $3 d$ also has complementarity to this probe. (B) RT-PCR showing the relative expression levels of mir390, the tas3 genes, and arf3a in wild-type and lb11-rgd1 apices. Loading and -RT controls are shown. $(C)$ Small RNA Northern showing that tasiR-ARF accumulates in wild-type, not in $1 b 11$ rgd1 apices. (D) 5' RACE analysis shows a single prominent arf3a cleavage product (arrowhead) only in wild type. RT-PCR of ubiquitin served as loading control. (E) Diagram of the arf3a transcript with tasiR-ARF complementary sites A and B. The $5^{\prime}$ ends of the tasiR-ARF cleavage products map to complementary site B. 
Moreover, these data indicate that $1 b 11$ forms an essential component of the ta-siRNA pathway and implicate this pathway in leaf polarity in maize.

Next, we analyzed the $1 b 11$ expression pattern by in situ hybridization to determine where in the vegetative apex ta-siRNA biogenesis may occur. $1 b 11$ expression is most pronounced in a dome of cells at the tip of the meristem that extends into the adaxial side of the initiating primordium (Fig. 4A). In the $\mathrm{P} 1$ and $\mathrm{P} 2$ primordia, $1 b 11$ is expressed preferentially on the adaxial side, and during later stages of leaf development, expression of $1 b 11$ becomes restricted to the margins and vasculature. This expression pattern suggests that ta-siRNAs may accumulate in a defined and perhaps polarized pattern in young leaves. We therefore analyzed the expression pattern of tasiR-ARF using in situ hybridization. In normal apices, hybridization to the tasiR-ARF complementary LNA probe (Fig. 3A) was most pronounced on the adaxial side of incipient and developing leaf primordia (Fig. 4B-D). However, some signal was detectable in $1 b 11$-rgd1 primordia (Fig. 4E). Because tas3 precursors, unlike miRNA precursors, are quite abundant, this suggests that the expression pattern in wild-type apices may reflect the accumulation of tas 3 transcripts, in addition to the mature tasiR-ARF. The persistence of tas3 transcripts in 1b11-rgd1 (Figs. 3B, 4E) suggests that their expression is regulated independently of the ta-siRNA pathway. More importantly, the expression pattern in wild-type apices suggests that tasiR-ARF and perhaps tas3 act foremost on the adaxial side of the developing primordia.

Considering the mechanism of ta-siRNA action, such polar expression suggests that the ta-siRNA pathway contributes to leaf polarity in part by restricting the accumulation of abaxial determinants, such as miR166, in a domain of the incipient primordium where adaxialabaxial polarity is established. To test this possibility, we analyzed the effect of loss of $1 b 11$ activity on expression of arf3a and the mir166 genes in the SAM. The low abundance of miRNA precursors precludes expression analyses of individual mir166 genes by in situ hybridization (Supplementary Fig. 1; Juarez et al. 2004a; Kidner and Timmermans 2006). We therefore developed laser capture microdissection (LCM) in combination with RT-PCR to analyze expression of these genes in the apex of wild-type and $1 b l 1$ mutants with high sensitivity and tissue-specific resolution. Cells were captured from the SAM plus the adaxial side of the incipient leaf and, to assess the role of the ta-siRNA pathway during primordium development, from the P1 plus P2 leaves (Fig. 4F). arf $3 a$ transcripts are undetectable in the wild-type SAM, but $\operatorname{arf} 3 a$ is expressed in the SAM of 1b11-rgd1. Transcript levels of arf3a are also increased in young leaf primordia of $1 b 11-$ rgd1 compared with wild type. This confirms that $1 b 11$ and the ta-siRNA pathway function to restrict the expression domain of arf3a in the SAM, as well as during primordium development. Of the mir166 genes that are differentially expressed between wild-type and $1 b 11$ apices (Fig. 1D), primary transcripts for mir166c accumulate specifically in the SAM and young leaf primordia of $1 b 11-r g d 1$, and expression levels for mir166i are increased in these mutant tissues (Fig. 4F). This indicates that the ectopic expression of miR166 in 1b11-rgd1 incipient primordia results from the broadened expression of mir166c and/or mir166i. Moreover, these data support the notion that the ta-siRNA pathway specifies adaxial

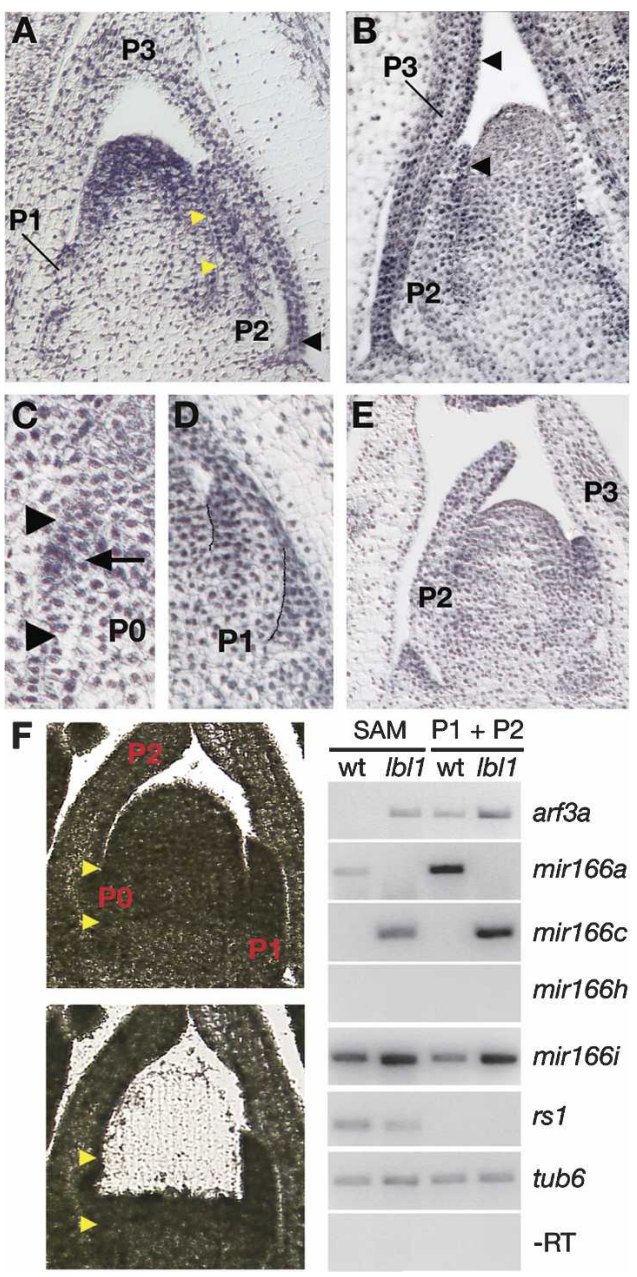

Figure 4. The ta-siRNA pathway acts on the adaxial side of developing leaf primordia and restricts expression of abaxial determinants. (A) Longitudinal section through a wild-type apex showing $1 b 11$ expression in the meristem tip and adaxially in leaf primordia The yellow arrowheads mark the position of the incipient or PO primordium, and the black arrowhead indicates expression at the $\mathrm{P} 3$ margin. $(B-E)$ In situ hybridization with the tasiR-ARF complementary LNA-modified DNA probe in longitudinal sections through wild-type $(B-D)$ and $1 b 11-r g d 1(E)$ apices. $(B)$ In wild type, tasiR-ARF accumulates predominantly on the adaxial side of developing leaves. Arrowheads highlight adaxial expression in $\mathrm{P} 2$ and $\mathrm{P} 3$ primordia. $(C)$ A close-up of a distinct incipient leaf shows adaxial tasiR-ARF expression (arrow). (D) A close-up of a P1 primordium also shows adaxial accumulation of tasiR-ARF. (E) Some hybridization to the tasiR-ARF complementary LNA probe is observed in $1 b 11$-rgd1, perhaps reflecting hybridization to tas 3 precursor transcripts. $(F$, left panels) lbl1-rgd1 apices before and after laser capture microdissection. The incipient leaf is marked by yellow arrowheads. (Right panel) RT-PCR analysis on LCM samples of the SAM or P1 plus P2 leaf primordia showing the effect of $1 b 11$-rgd1 on expression of $a r f 3 a$ mir166a, mir166c, and mir166i. The SAM-specific homeobox gene rough sheath1 (rs1) was analyzed as LCM control (Schneeberger et al. 1995). Loading and -RT controls are also shown.

fate by spatially defining the expression domains of these mir166 genes, although perhaps indirectly via regulation of ARF3 transcription factors or other abaxial determinants.

Recent studies have shown that tasiR-ARF-mediated cleavage of $A R F 3$ is required for normal leaf development in Arabidopsis, and accordingly, infer a role for the 
ta-siRNA pathway in leaf polarity (Fahlgren et al. 2006; Garcia et al. 2006). However, the contribution of this pathway to adaxial-abaxial patterning in Arabidopsis remains unclear. In fact, $A R F 3$ is expressed uniformly throughout young leaf primordia (Pekker et al. 2005), and Arabidopsis mutants that block the biogenesis of ta-siRNAs develop no obvious leaf polarity defects (Peragine et al. 2004; Adenot et al. 2006). Moreover, the distribution of trichomes in such mutants as well as in plants expressing a tasiR-ARF-insensitive allele of $A R F 3$ is inconsistent with the predicted abaxializing phenotype (Fahlgren et al. 2006; Hunter et al. 2006).

Our findings uncover two small RNAs, miR390 and tasiR-ARF, that act upstream of previously known components in the leaf polarity pathway. tasiR-ARF accumulates on the adaxial site of leaf primordia and contributes to organ polarity by spatially restricting the expression domain of abaxial determinants, including miR166, in the incipient and young leaves. This presents the intriguing possibility that distinct small regulatory RNAs direct the establishment of opposing domains along the adaxial-abaxial axis; whereas tasiR-ARF defines the adaxial domain, miR166 delineates the abaxial domain by restricting expression of the adaxializing $H D$-ZIPIII genes. These findings thus reveal a novel patterning mechanism in development.

A function for tasiR-ARF and miR166 in the incipient leaf also raise the question of whether small RNAs contribute to the production or perception of positional information from the SAM required for adaxial-abaxial patterning (Sussex 1951; Reinhardt et al. 2005). tasiRARF and miR166 are expressed in essentially complementary domains in the incipient as well as young leaf primordia, consistent with the proposed model that the ta-siRNA pathway, perhaps via arf3 or other abaxial determinants, spatially restricts the accumulation of miR166 in these tissues (Supplementary Fig. 3). Based on the graded pattern of miR166 accumulation in developing leaf primordia, we previously suggested that a mobile signal controls expression of mir166 precursors or that miR166 itself acts as a local mobile signal (Juarez et al. 2004a). The loss of this graded polar pattern of expression in $1 b 11$ mutants and the involvement of the ta-siRNA pathway in the spatial regulation of abaxial determinants is therefore particularly intriguing. The non-cellautonomous nature of gene silencing is mediated by a distinct branch of the RNA interference (RNAi) machinery (Dunoyer et al. 2005). Ta-siRNA biogenesis utilizes components of this unique RNAi pathway, presenting the possibility that the ta-siRNA pathway may similarly include a non-cell-autonomous component. The unique properties of ta-siRNA biogenesis might also exist to limit tasiR-ARF production, as mature tasiR-ARFs scarcely accumulate despite an abundance of precursor transcripts (Allen et al. 2005; Lu et al. 2006). Their low dose would implicitly affect their efficacy (Bartel 2004; Jones-Rhoades et al. 2006) and, if tasiR-ARFs are themselves able to move, their range of activity (Voinnet 2005). Both may be important variables if a balance between adaxial and abaxial fates is to be struck.

\section{Materials and methods}

Cloning of lbl1

$1 b 11$ was cloned using a directed transposon-tagging approach. Please see Supplemental Material for details. Full-length $1 b 11$ cDNA clones were obtained by RT- and RACE-PCR using total RNA from apices of 2-wkold seedlings. Mutant $1 b 11$ alleles were sequenced from RT-PCR products amplified using gene-specific primers that spanned the full-length $1 b 11$ cDNA.

\section{Sequence analysis}

Sequences corresponding to the maize tas 3 and mir390 loci were assembled using EST and genomic (http://magi.plantgenomics.iastate.edu) databases. Potential ta-siRNA targets were identified in the maize EST data set following the criteria established in Allen et al. (2005). Gene predictions were verified by RT-PCR, RACE, and sequence analysis. The GenBank accession number for $1 b 11$ is DQ832257, and the MAGIv4 accession numbers are mir390, 106773; tas $3 a, 69518$; tas $3 b, 65670$; tas $3 c$, 63185; tas3d, PUIFI48.F; and arf3a, 48978.

\section{Molecular biology}

RNA from vegetative apices including the SAM and four leaf primordia was isolated and analyzed by RT-PCR as described (Juarez et al. 2004b). 5' RACE was performed as described (Allen et al. 2005). Primer sequences and detailed PCR conditions for all genes analyzed are available upon request. Low-molecular-weight RNA (5 $\mu \mathrm{g})$ from vegetative apices was extracted, blotted, and hybridized with an end-labeled 16-nt LNA-modified oligonucleotide probe (Exiqon) complementary to tasiR-ARF (CTTA CAAGGTCAAGAA) as described (Allen et al. 2005).

In situ hybridization

In situ hybridizations were performed as described (Kidner and Timmermans 2006). The tasiR-ARF LNA probe was hybridized at $37^{\circ} \mathrm{C}$. The $r l d 2$ and $1 b 11$ probes comprises nucleotides 625-1677 and 120-810 of the coding sequence, respectively, and were used at a concentration of $0.5 \mathrm{ng}$ $\mu \mathrm{L}^{-1} \mathrm{~kb}^{-1}$. miR166 probes are as described (Juarez et al. 2004a).

LCM and expression analysis

Shoot apices of 2-wk-old seedlings were embedded as described (http:// maize-meristems.plantgenomics.iastate.edu/resources/protocols). Six independent apices were captured for wild-type and $1 b 11$ samples. LCM was performed using a PALM MicroBeam system. RNA samples were isolated using the PicoPure RNA isolation kit (Arcturus) and amplified using the RiboAmpHS RNA Amplification kit (Arcturus), according to the manufacturer's instructions. RT-PCR was performed on 50-150 ng of amplified, DNase-treated aRNA using the OneStep RT-PCR kit (Qiagen). Primer sequences and PCR conditions are available upon request.

\section{Acknowledgments}

We thank Julie Thomas, Krista Marran, and Amanda Grieco for excellent technical assistance; Tim Mulligan for plant care; and Cris Kuhlemeier, Greg Hannon, and members of the Timmermans laboratory for critical reading of the manuscript. This work was supported by grants to M.T. from the USDA (03420) and the NSF (0315676). F.N. was funded in part by a CSHL Association fellowship, S.M. and the LCM experiments were funded by NSF VCA-PGR 0321595, and D.C. is an NSF graduate research fellow and a G. and M. Matheson fellow.

\section{References}

Adenot, X., Elmayan, T., Lauressergues, D., Boutet, S., Bouche, N., Gasciolli, V., and Vaucheret, H. 2006. DRB4-dependent TAS3 trans-acting siRNAs control leaf morphology through AGO7. Curr. Biol. 16: 927-932.

Allen, E., Xie, Z., Gustafson, A., and Carrington, J. 2005. microRNAdirected phasing during trans-acting siRNA biogenesis in plants. Cell 121: $207-221$.

Alvarez-Garcia, I. and Miska, E. 2005. MicroRNA functions in animal development and human disease. Development 132: 4653-4662.

Bartel, D. 2004. MicroRNAs: Genomics, biogenesis, mechanism, and function. Cell 116: 281-297.

Dunoyer, P., Himber, C., and Voinnet, O. 2005. DICER-LIKE 4 is required for RNA interference and produces the 21-nucleotide small interfering RNA component of the plant cell-to-cell silencing signal. Nat. Genet. 37: 1356-1360.

Emery, J.F., Floyd, S.K., Alvarez, J., Eshed, Y., Hawker, N., Izhaki, A., 
Baum, S.F., and Bowman, J.L. 2003. Radial patterning of Arabidopsis shoots by class III HD-ZIP and KANADI genes. Curr. Biol. 13: 17681774

Fahlgren, N., Montgomery, T., Howell, M., Allen, E., Dvorak, S., Alexander, A., and Carrington, J. 2006. Regulation of AUXIN RESPONSE FACTOR3 by TAS3 ta-siRNA affects developmental timing and patterning in Arabidopsis. Curr. Biol. 16: 939-944.

Garcia, D., Collier, S., Byrne, M., and Martienssen, R. 2006. Specification of leaf polarity in Arabidopsis via the trans-acting siRNA pathway. Curr. Biol. 16: 933-938.

Gasciolli, V., Mallory, A., Bartel, D., and Vaucheret, H. 2005. Partially redundant functions of Arabidopsis DICER-like enzymes and a role for DCL4 in producing trans-acting siRNAs. Curr. Biol. 15: 14941500.

Hunter, C., Willmann, M., Wu, G., Yoshikawa, M., de la Luz GutierrezNava, M., and Poethig, S. 2006. Trans-acting siRNA-mediated repression of ETTIN and ARF4 regulates heteroblasty in Arabidopsis. Development 133: 2973-2981.

Jones-Rhoades, M., Bartel, D., and Bartel, B. 2006. MicroRNAs and their regulatory roles in plants. Annu. Rev. Plant Biol. 57: 19-53.

Juarez, M., Kui, J., Thomas, J., Heller, B., and Timmermans, M. 2004a. microRNA-mediated repression of rolled leaf1 specifies maize leaf polarity. Nature 428: $84-88$.

Juarez, M., Twigg, R., and Timmermans, M. 2004b. Specification of adaxial cell fate during maize leaf development. Development 131: 4533-4544.

Kidner, C. and Martienssen, R. 2004. Spatially restricted microRNA directs leaf polarity through ARGONAUTE1. Nature 428: 81-84.

Kidner, C. and Timmermans, M. 2006. In situ hybridization as a tool to study the role of microRNAs in plant development. Methods Mol. Biol. 342: 159-179.

Lu, C., Kulkarni, K., Souret, F., Muthu, V., Tej, S., Poethig, R., Henderson, I., Jacobsen, S., Wang, W., Green, P., et al. 2006. MicroRNAs and other small RNAs enriched in the Arabidopsis RNA-dependent RNA polymerase-2 mutant. Genome Res. 16: 1276-1288.

McConnell, J., Emery, J., Eshed, Y., Bao, N., Bowman, J., and Barton, M. 2001. Role of PHABULOSA and PHAVOLUTA in determining radial patterning in shoots. Nature 411: 709-713.

Mourrain, P., Beclin, C., Elmayan, T., Feuerbach, F., Godon, C., Morel, J., Jouette, D., Lacombe, A., Nikic, S., Picault, N., et al. 2000. Arabidopsis SGS2 and SGS3 genes are required for posttranscriptional gene silencing and natural virus resistance. Cell 101: 533-542.

Pekker, I., Alvarez, J., and Eshed, Y. 2005. Auxin response factors mediate Arabidopsis organ asymmetry via modulation of KANADI activity. Plant Cell 17: 2899-2910.

Peragine, A., Yoshikawa, M., Wu, G., Albrecht, H., and Poethig, S. 2004. $S G S 3$ and SGS2/SDE1/RDR6 are required for juvenile development and the production of trans-acting siRNAs in Arabidopsis. Genes \& Dev. 18: 2368-2379.

Reinhardt, D., Frenz, M., Mandel, T., and Kuhlemeier, C. 2005. Microsurgical and laser ablation analysis of leaf positioning and dorsoventral patterning in tomato. Development 132: 15-26.

Schneeberger, R., Becraft, P., Hake, S., and Freeling, M. 1995. Ectopic expression of the knox homeobox gene rough sheath1 alters cell fate in the maize leaf. Genes \& Dev. 9: 2292-2304.

Sussex, I. 1951. Experiments on the cause of dorsiventrality in leaves. Nature 167: 651-652.

Timmermans, M., Schultes, N., Jankovsky, J., and Nelson, T. 1998. Leafbladeless1 is required for dorsoventrality of lateral organs in maize. Development 125: 2813-2823.

Timmermans, M., Juarez, M., and Phelps-Durr, T. 2004. A conserved microRNA signal specifies leaf polarity. Cold Spring Harb. Symp. Quant. Biol. 69: 409-417.

Vazquez, F., Vaucheret, H., Rajagopalan, R., Lepers, C., Gasciolli, V., Mallory, A., Hilbert, J., Bartel, D., and Crete, P. 2004. Endogenous trans-acting siRNAs regulate the accumulation of Arabidopsis mRNAs. Mol. Cell 16: 69-79.

Voinnet, O. 2005. Non-cell autonomous RNA silencing. FEBS Lett. 579: $5858-5871$

Williams, L., Carles, C., Osmont, K., and Fletcher, J. 2005. A database analysis method identifies an endogenous trans-acting short-interfering RNA that targets the Arabidopsis ARF2, ARF3, and ARF4 genes. Proc. Natl. Acad. Sci. 102: 9703-9708.
Xie, Z., Allen, E., Wilken, A., and Carrington, J. 2005. DICER-LIKE 4 functions in trans-acting small interfering RNA biogenesis and vegetative phase change in Arabidopsis thaliana. Proc. Natl. Acad. Sci. 102: 12984-12989.

Yoshikawa, M., Peragine, A., Park, M., and Poethig, S. 2005. A pathway for the biogenesis of trans-acting siRNAs in Arabidopsis. Genes \& Dev. 19: 2164-2175. 


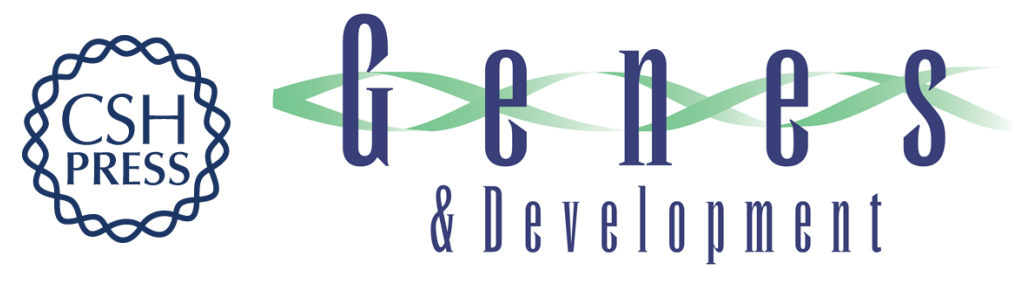

\section{Two small regulatory RNAs establish opposing fates of a developmental axis}

Fabio T.S. Nogueira, Shahinez Madi, Daniel H. Chitwood, et al.

Genes Dev. 2007, 21:

Access the most recent version at doi:10.1101/gad.1528607

\section{Supplemental http://genesdev.cshlp.org/content/suppl/2007/03/19/21.7.750.DC1 Material}

References This article cites 30 articles, 12 of which can be accessed free at: http://genesdev.cshlp.org/content/21/7/750.full.html\#ref-list-1

\section{License}

Email Alerting

Receive free email alerts when new articles cite this article - sign up in the box at the top Service 